\title{
A circulating miRNA signature for early diagnosis of acute kidney injury following acute myocardial infarction
}

\author{
Pei-Chun Fan ${ }^{1,2 \dagger}$, Chia-Chun Chen ${ }^{3,4 \dagger}$, Chen-Ching Peng 3 , Chih-Hsiang Chang 1,2, Chia-Hung Yang ${ }^{5}$, Chi Yang ${ }^{3}$, \\ Lichieh Julie $\mathrm{Chu}^{3}$, Yung-Chang Chen ${ }^{6}$, Chih-Wei Yang ${ }^{1}$, Yu-Sun Chang ${ }^{3}$ and Pao-Hsien Chu ${ }^{7^{*}}$ (1)
}

\begin{abstract}
Background: Acute kidney injury (AKI) is a common complication of acute myocardial infarction (AMI), and is associated with adverse outcomes. The study aimed to identify a miRNA signature for the early diagnosis of post-AMI AKI.

Methods: A total of 108 patients admitted to a coronary care unit (CCU) were divided into four subgroups: $\mathrm{AMI}^{-} \mathrm{AKI}^{-}$, $\mathrm{AMI}^{+} \mathrm{AKI}^{-}, \mathrm{AMI}^{+} \mathrm{AKI}^{+}$, and $\mathrm{AMI}^{-} \mathrm{AKI}{ }^{+}$. Thirty-six miRNA candidates were selected based on an extensive literature review. Real-time quantitative RT-PCR analysis was used to determine the expression levels of these miRNAs in the serum collected on the day of CCU admittance. TargetScan 7.1 and miRDB databases were used for target prediction and Metacore 6.13 was used for pathway analysis.

Results: Through a stepwise selection based on abundance, hemolytic effect and differential expression between four groups, 9 miRNAs were found to have significantly differential expression levels as potential biomarkers for post-AMI AKI specifically. Noticeably, the expression levels of miR-24, miR-23a and miR-145 were significantly downregulated in $\mathrm{AMI}^{+} \mathrm{AKI}^{+}$patients compared to those in $\mathrm{AMI}^{+} \mathrm{AKI}^{-}$patients. Combination of the three miRNAs as a panel showed the best performance in the early detection of AKI following AMI (AUC $=0.853$, sensitivity 95.65\%), compared to the analysis of serum neutrophil gelatinase-associated lipocalin (AUC $=0.735$, sensitivity 63.16\%). Furthermore, bioinformatic analysis indicated that these three miRNAs regulate the transforming growth factor beta signaling pathway and involve in apoptosis and fibrosis in AKI.
\end{abstract}

Conclusions: For the first time, this study identify a unique circulating miRNA signature (miR-24-3p, miR-23a-3p, miR145-5p) that can potentially early detect AKI following AMI and may be involved in renal injury and fibrosis in postAMI AKI pathogenesis.

Keywords: MicroRNAs, Acute kidney injury, Acute myocardial infarction, TGF- $\beta$

\section{Background}

Acute kidney injury (AKI) is a common and important complication of various critical illnesses [1]. Among them, the incidence of AKI following acute myocardial infarction (AMI) is approximately $12-37 \%$ [2-5].

\footnotetext{
*Correspondence: taipei.chu@gmail.com

${ }^{\dagger}$ Pei-Chun Fan and Chia-Chun Chen contributed equally to the manuscript

${ }^{7}$ Department of Cardiology, Chang Gung Memorial Hospital, Chang Gung University College of Medicine, 199 Tung Hwa North Road,

Taipei 105, Taiwan

Full list of author information is available at the end of the article
}

Compared to those without AKI, patients with AMI who develop incident AKI have significantly prolonged hospital stays and increased rates of hospital mortality (14-39\% vs. $0.5-9 \%)$ and 1-year mortality (16-50\% vs. $5-14 \%)[2-4,6,7]$. In the short term, AKI can lead to uremic symptoms, fluid overload, electrolyte imbalance, metabolic acidosis, coagulopathy and increased risk of infection. In addition, the severity and duration of AKI are correlated with the risk of chronic kidney disease and end-stage renal disease, leading to increased economic, social and personal burdens (Additional file 1: Figure S1a) $[8,9]$. 
Although research advances have been made in recent decades, a definite and effective treatment for AKIis still lacking. The best strategies currently focus on prevention, early diagnosis and early interventions aiming at managing the underlying etiologies and complications of AKI. The widely accepted diagnostic criteria for AKI are based on changes of serum creatinine (SCr) and urine output; they include the Risk, Injury, Failure, Loss of kidney function, and End-stage kidney disease (RIFLE) classification [1] the Acute Kidney Injury Network (AKIN) criteria [10] and the Kidney Disease Improving Global Outcomes (KDIGO) criteria [11]. However, obvious changes in $\mathrm{SCr}$ may not be seen until 48-72 h after renal insult, potentially delaying the diagnosis of AKI. To enable the early diagnosis of AKI, researchers have investigated various novel biomarkers, such as neutrophil gelatinase-associated lipocalin (NGAL) [12] and kidney injury molecule-1 (KIM-1) [13]. However, these biomarkers may be interfered by diseases other than AKI, and there are controversies regarding cut-offs. To date, researchers have been unable to establish a biomarker-guided clinical strategy that reliably improves the clinical outcome in patients with AKI.

MicroRNAs (miRNAs) are endogenous single-stranded noncoding mRNAs of 19-23 nucleotides that play critical roles in the post-transcriptional regulation of multiple biological cell functions, including proliferation, differentiation, metabolism and apoptosis [14]. Accumulating evidence suggests that certain miRNAs are up- or downregulated in response to AKI and AMI, respectively. These miRNAs have been suggested as contributing to the pathogeneses of AKI and AMI and as potential biomarkers for both diseases $[15,16]$. Nevertheless, no previous study has explored these related miRNA expression levels and functions in post-AMI AKI, and the interaction of miRNAs and their downstream targets also have not been completely elucidated. Furthermore, miRNAs have emerged as promising therapeutic targets in various diseases, including kidney fibrosis and diabetic kidney disease [17], with many ongoing clinical trials. However, the therapeutic implication of miRNAs in AKI has not yet been explored.

This study aims to identify a circulating miRNA signature for post-AMI AKI, in the hopes of facilitating early diagnosis and prompt early intervention. Such miRNAs could also serve as potential therapeutic targets to improve clinical outcomes in patients with post-AMI AKI.

\section{Methods}

\section{Study design and patient cohort}

The study design was summarized in Additional file 1: Figure S1. The study was performed in the coronary care unit (CCU) of a tertiary care referral center. The patients admitted in this CCU from 2010 to 2014 were prospectively enrolled. Patients less than 18 years old, patients who had previously received a kidney transplant, and patients with end-stage renal disease were excluded. The enrolled patients were classified into four groups: $\mathrm{AMI}^{-} \mathrm{AKI}^{-}, \mathrm{AMI}^{+} \mathrm{AKI}^{-}, \mathrm{AMI}^{+} \mathrm{AKI}^{+}$and $\mathrm{AMI}^{-} \mathrm{AKI}^{+}$. Demographic characteristics, causes of admission, laboratory data, and hospital outcomes were prospectively collected. The diagnosis of AMI was based on the Consensus Conference criteria for the universal definition of myocardial infarction [18]. AKI was defined based on a change in SCr, as listed in the KDIGO criteria [11]. The study protocol was approved by the local institutional review board, and informed consents were obtained from all participants.

\section{Sample collection, hemolysis detection, and serum NGAL analysis}

Blood samples were collected in non-heparinized tubes within $24 \mathrm{~h}$, at $48 \mathrm{~h}$ and at 1 week of admission. Blood samples were centrifuged at $2000 \mathrm{rpm}$ for $10 \mathrm{~min}$, and the obtained serum was stored at $-80{ }^{\circ} \mathrm{C}$ for further processing. Serum levels of NGAL were measured in duplicate using commercially available enzyme-linked immunosorbent assay (ELISA) kits according to the manufacturer's instructions (DLCN20; R\&D Systems, Minneapolis, MN, USA). When a measurement exhibited a $>5 \%$ variance, a third analysis was performed to ensure a variance of $\leq 5 \%$. For the purpose of early diagnosis, only samples at the first time point (within $24 \mathrm{~h}$ ) were used to miRNA detection. Before RNA extraction, hemolysis was directly determined in $2 \mu \mathrm{L}$ serum samples by detecting the absorbance of hemoglobin at $414 \mathrm{~nm}$ using the NanoDrop 2000c UV-Vis spectrophotometer (Thermo Scientific, DE, USA).

\section{Selection of 36 miRNA candidates as potential biomarkers from literature review}

A thorough and critical literature review of 119 research articles related to AKI, AMI and cardiovascular diseases (CVD) was performed (Additional file 2: Tables S1, S2). The total reference counts and the numbers of studies related to AKI, AMI and CVD for each miRNA are summarized in Additional file 1: Figure S2a. It is worth noting that many of these miRNAs overlapped in the cardiac and renal diseases. Among the most frequently cited miRNAs, miR-21 was the top one among the 15 miRNAs commonly reported in all three disease types (Additional file 1: Figure S2b). In the 119 studies, miRNAs were detected in various sample types (liquid or tissue biopsies) and species origins (human or murine); the distribution of sample types grouped by disease is shown 
in Additional file 1: Figure S2c. About 68.2\% (30/44) and 66.7\% (62/93) of the miRNAs studied in AMI and CVD were tested in patient blood or tissue samples, whereas less studies $(27.3 \%, 24 / 88)$ of those studied in AKI were tested in blood, tissue or urine samples. From the compiled data, 36 highly reported miRNAs as the miRNA candidate set were selected.

\section{RNA extraction and heparinase treatment}

An aliquot of $250 \mu \mathrm{L}$ serum samples were mixed with $1000 \mu \mathrm{L}$ QIAZol Lysis Reagent (Qiagen) and a spiked-in control consisting of $10^{7}$ copies of synthetic RNA corresponding to Caenorhabditis elegans miR-39 (5'-UCA CCGGGUGUAAAUCAGCUUG) (IDT, Coralville, IA, USA). Total RNA was extracted from this mixture using a miRNeasy Mini Kit (Qiagen) according to the manufacturer's instructions. The purified RNA was eluted in $30 \mu \mathrm{L}$ nuclease-free water and stored at $-80{ }^{\circ} \mathrm{C}$ until use. To avoid the heparin-related interference, $0.5 \mathrm{U}$ heparinase I (Sigma-Aldrich, MO, USA) was added to the prereverse transcription (RT) mixture containing $5.4 \mu \mathrm{L}$ serum RNA, $2 \mathrm{U} / \mu \mathrm{L}$ RNase out (Invitrogen), $1 \times$ PCR buffer (Roche Diagnostics) and $1.25 \mathrm{mM} \mathrm{MgCl}_{2}$, and the samples were incubated at $25^{\circ} \mathrm{C}$ for $1 \mathrm{~h}$.

\section{Reverse transcription and quantitative polymerase chain reaction}

The multiplex RT-qPCR of 37 miRNAs was performed as described previously [19]. The resulting Ct value of each miRNA was first normalized with respect to that of the spiked-in cel-miR-39 by adjusting the miRNA Ct values with the cel-miR-39 ratio (a ratio calculated based on the differences of cel-miR-39 Ct value in each sample to cel-miR-39 median of total samples). To adjust for variations in the total RNA amounts of individual samples, the cel-miR-39-normalized Ct value was further normalized to each individual RNA concentration, which was determined using a NanoDrop 3300 Fluorospectrophotometer (Thermo Scientific, DE, USA) and a Quant-iT ${ }^{\mathrm{TM}}$ RiboGreen ${ }^{\circledR}$ RNA Assay (Invitrogen). MiRNAs with normalized Ct values over 40 were defined as 'undetectable.' Relative miRNA expression levels were represented as 40-Ct.

\section{miRNA target prediction and pathway analysis}

The MiRDB and TargetScan 7.1 databases were used to identify potential human miRNA target genes. The predicted target genes were subjected to Metacore 6.13 pathway map analysis.

\section{Statistical methods}

Continuous variables were summarized as the mean \pm standard error and percentages. Normally distributed continuous variables were compared by the Student's t-test or Fisher's exact test, and non-normally distributed ones were compared by the MannWhitney U test. Categorical variables were compared using the $x^{2}$ test or Fisher's exact test. Correlation coefficients were calculated using Spearman's rank correlation analysis between groups. Non-parametric Kruskal-Wallis test with post hoc Dunn's tests were conducted to analyze the differences among all groups. The area under the receiver operating characteristic curve (AUC) was used to determine the discriminatory power, and the Youden's index was used to assess the optimal cut-off value. Algorithms of two- or threemiRNAs panels were built by logistic regression and are, respectively, $\operatorname{logit}[\operatorname{Pr}(\mathrm{Y}=1)]=13.962+0.982$ $\times$ miR-23a-3p $-1.987 \times$ miR-24-3p and logit $[\operatorname{Pr}(\mathrm{Y}=1)]$ $=17.12+0.742 \times$ miR-23a-3p $-2.558 \times$ miR-24-3p + $0.772 \times \mathrm{miR}-145-5 \mathrm{p}$. All statistical tests were 2-tailed, and $P<0.05$ was considered statistically significant. All statistical analyses were conducted using the IBM SPSS Statistics 22 (SPSS Inc., IL, USA). Plots were graphed using Prism 5 (GraphPad).

\section{Results}

\section{Patient characteristics}

As shown in Table 1, a total of 108 patients were enrolled for analysis and the $\mathrm{AMI}^{-} \mathrm{AKI}^{-}, \mathrm{AMI}^{+} \mathrm{AKI}^{-}, \mathrm{AMI}^{+} \mathrm{AKI}^{+}$ and $\mathrm{AMI}^{-} \mathrm{AKI}^{+}$subgroups contained 30, 26, 23, and 29 patients, respectively. All four groups have similar gender distribution, body weight, white blood cell count, serum level of alanine aminotransferase, mean arterial pressure, ejection fraction and in-hospital mortality. Compared to $\mathrm{AMI}^{+} \mathrm{AKI}^{-}$patients, $\mathrm{AMI}^{+} \mathrm{AKI}^{+}$patients had an older age and higher prevalences of pre-existing hypertension and congestive heart failure. The average $\mathrm{SCr}$ values in $\mathrm{AMI}^{+} \mathrm{AKI}^{+}$and $\mathrm{AMI}^{+} \mathrm{AKI}^{-}$patients were $3.4 \pm 0.5$ and $0.9 \pm 0.1 \mathrm{mg} / \mathrm{dL}$, respectively. The average serum NGAL levels in $\mathrm{AMI}^{+} \mathrm{AKI}^{+}$and $\mathrm{AMI}^{+} \mathrm{AKI}^{-}$patients were $140.1 \pm 24.4$ and $64.3 \pm 7.5 \mathrm{ng} / \mathrm{dL}$, respectively. Compared to $\mathrm{AMI}^{+} \mathrm{AKI}^{-}$patients, the $\mathrm{AMI}^{+} \mathrm{AKI}^{+}$patients had lower hemoglobin, lower serum albumin, higher troponin-I and higher B-type natriuretic peptide. Three (13\%) of the $\mathrm{AMI}^{+} \mathrm{AKI}^{+}$patients required renal replacement therapy. Three $(13 \%)$ of the $\mathrm{AMI}^{+} \mathrm{AKI}^{+}$patients died during admission.

miR-24, miR-23a and miR-145 are the most promising targets for the early detection of post-AMI AKI

A work flow diagram for our identification of the clinically applicable miRNAs with the best discriminatory power in detecting post-AMI AKI is shown in Fig. 1. To increase data reliability, the five least abundant miRNAs 
Table 1 Demographic data and clinical characteristics of the study population

\begin{tabular}{|c|c|c|c|c|c|c|}
\hline Group & $\begin{array}{l}\mathrm{AMI}^{-} \mathrm{AKI}^{-} \\
(\mathrm{n}=30)\end{array}$ & $\begin{array}{l}\mathrm{AMI}^{+} \mathrm{AKI}^{-} \\
(\mathrm{n}=26)\end{array}$ & $\begin{array}{l}\mathrm{AMI}^{+} \mathrm{AKI}^{+} \\
(\mathrm{n}=23)\end{array}$ & $\begin{array}{l}\mathrm{AMI}^{-} \mathrm{AKI}^{+} \\
(\mathrm{n}=29)\end{array}$ & $\begin{array}{l}P \text {-value } \\
\text { (four groups) }\end{array}$ & $\begin{array}{l}P \text {-value } \\
\left(\mathrm{AMI}^{+} \mathrm{AKI}^{-} \mathrm{vs}\right. \\
\left.\mathrm{AMI}^{+} \mathrm{AKI}^{+}\right)\end{array}$ \\
\hline Age (years) & $61 \pm 3$ & $59 \pm 2$ & $73 \pm 2$ & $71 \pm 2$ & $<0.001$ & $<0.001$ \\
\hline Sex, male (\%) & $19(63.3)$ & $22(84.6)$ & $19(82.6)$ & $19(65.5)$ & 0.165 & 0.576 \\
\hline Body weight (kg) & $64.5 \pm 1.9$ & $68.9 \pm 2.6$ & $65.3 \pm 2.3$ & $64.9 \pm 2.6$ & 0.563 & 0.321 \\
\hline Diabetes mellitus (\%) & $7(23.3)$ & $7(26.9)$ & $12(52.2)$ & $16(55.2)$ & 0.023 & 0.064 \\
\hline Hypertension (\%) & $14(46.7)$ & $14(53.8)$ & $20(87.0)$ & $23(79.3)$ & 0.004 & 0.013 \\
\hline Coronary artery disease (\%) & $8(26.7)$ & $26(100.0)$ & $23(100.0)$ & $8(27.6)$ & $<0.001$ & 1.000 \\
\hline Heart failure (\%) & $13(43.3)$ & $2(7.7)$ & $15(65.2)$ & $22(75.9)$ & $<0.001$ & $<0.001$ \\
\hline Blood urea nitrogen (mg/dL) & $15 \pm 1$ & $14 \pm 1$ & $40 \pm 6$ & $53 \pm 6$ & $<0.001$ & $<0.001$ \\
\hline Serum creatinine (mg/dL) & $0.8 \pm 0.1$ & $0.9 \pm 0.1$ & $3.4 \pm 0.5$ & $3.0 \pm 0.3$ & $<0.001$ & $<0.001$ \\
\hline Serum NGAL (ng/mL) & $72.0 \pm 9.0$ & $64.3 \pm 7.5$ & $140.1 \pm 24.4$ & $264.8 \pm 56.1$ & $<0.001$ & 0.007 \\
\hline WBC $\left(10^{3} / \mu \mathrm{L}\right)$ & $8.2 \pm 0.4$ & $10.4 \pm 0.6$ & $12.6 \pm 1.2$ & $11.7 \pm 2.0$ & 0.071 & 0.096 \\
\hline Hemoglobin (g/dL) & $13.0 \pm 0.4$ & $14.8 \pm 0.2$ & $12.2 \pm 0.5$ & $11.0 \pm 0.5$ & $<0.001$ & $<0.001$ \\
\hline Sugar (mg/dL) & $122 \pm 9$ & $171 \pm 18$ & $183 \pm 20$ & $175 \pm 15$ & 0.033 & 0.671 \\
\hline $\operatorname{ALT}(\mathrm{U} / \mathrm{L})$ & $33 \pm 6$ & $31 \pm 5$ & $47 \pm 11$ & $88 \pm 54$ & 0.565 & 0.222 \\
\hline Albumin (g/L) & $3.9 \pm 0.1$ & $3.9 \pm 0.1$ & $3.4 \pm 0.1$ & $3.3 \pm 0.1$ & $<0.001$ & $<0.001$ \\
\hline Troponin I (ng/mL) & $2.30 \pm 1.13$ & $3.43 \pm 1.47$ & $15.98 \pm 5.62$ & $0.83 \pm 0.27$ & 0.005 & 0.041 \\
\hline $\mathrm{BNP}(\mathrm{pg} / \mathrm{mL})$ & $386 \pm 112$ & $243 \pm 132$ & $1098 \pm 303$ & $1460 \pm 288$ & 0.001 & 0.038 \\
\hline Mean arterial pressure $(\mathrm{mmHg})$ & $87 \pm 3$ & $89 \pm 3$ & $90 \pm 3$ & $86 \pm 4$ & 0.759 & 0.804 \\
\hline Ejection fraction (\%) & $55 \pm 3$ & $58 \pm 3$ & $48 \pm 4$ & $50 \pm 4$ & 0.226 & 0.050 \\
\hline Renal replacement therapy & $0(0)$ & $0(0)$ & $3(13.0)$ & $4(13.8)$ & 0.021 & 0.096 \\
\hline In-hospital mortality & $1(3.3)$ & $0(0)$ & $3(13.0)$ & $2(6.9)$ & 0.237 & 0.096 \\
\hline
\end{tabular}

Values are mean \pm standard error

$A M I$ acute myocardial infarction, $A K I$ acute kidney injury, $A L T$ alanine aminotransferase, $B N P$ B-type natriuretic peptide, $B U N$ blood urea nitrogen, $N G A L$ neutrophil gelatinase-associated lipocalin, $R R T$ renal replacement therapy, $W B C$ white blood cell

(miR-200c, miR-205, miR-135a, miR-489 and miR-210), which were undetectable ( $\mathrm{Ct}$ value $>40)$ in more than $50 \%$ of our samples, were eliminated (Fig. 1a). In serum samples, hemolysis may cause miRNAs to be released from red blood cells, substantially altering the circulating miRNA content [20]. To exclude the miRNAs whose levels may be altered by hemolysis, the serum samples were separated into non-hemolytic $(n=28)$ and hemolytic $(\mathrm{n}=80)$ groups by their $\mathrm{OD}_{414 \mathrm{~nm}}$ values of hemoglobin, and then compared miRNA expression between the groups. As shown in Fig. 1b, the expression levels of 14 of 31 miRNAs were significantly increased in hemolytic samples. Among them, miR-451a showed that largest alteration, with a 4.3-fold change in hemolytic samples; this finding is consistent with many previous reports [21-23]. Finally, the remaining 17 miRNA candidates were compared among all 4 groups by Kruskal-Wallis test. Among them, 15 miRNAs were significantly altered between these four groups (Additional file 1: Table S3). Noticeably, after multiple comparisons, 9 of them had significantly differential expression in AMI patients who developed AKI compared to those without developed AKI (Fig. 1c and Additional file 2: Table S1). However, these miRNAs were not altered between $\mathrm{AMI}^{-} \mathrm{AKI}^{+}$and $\mathrm{AMI}^{-} \mathrm{AKI}^{-}$group and these data disclosed them specifically as potential biomarkers for post-AMI AKI. In order to facilitate clinical practice with minimum miRNA targets, the top 3 highly abundant miRNAs (miR-24, miR23a and miR-145)were selected as the most potential biomarkers for further evaluating (Fig. 1c, d).

The expression levels of miR-24, miR-23a and miR145were significantly down-regulated in $\mathrm{AMI}^{+} \mathrm{AKI}^{+}$ patients compared to $\mathrm{AMI}^{+} \mathrm{AKI}^{-}$patients (Fig. 2a; 0.27 -, 0.32- and0.34-fold, respectively). For discrimination ability, the AUC values of miR-24 and miR-23a were greater than 0.8 , and all three miRNAs had better performances than that of serum NGAL (AUC $=0.735$ ). Using the cut-off values obtained from Youden's index on the ROC curves of miRNAs and serum NGAL, sensitivities ranging from 60.87 to $86.96 \%$ and specificities of $53.85-92.31 \%$ were obtained (Table 2). The sensitivity of miR-23a was very close to that of serum NGAL; however, the sensitivities of miR-24 and miR-145 were greatly improved (by $\sim 20 \%$ ). These results strongly suggestedt hat the selected miRNAs may have potential for clinical application. 


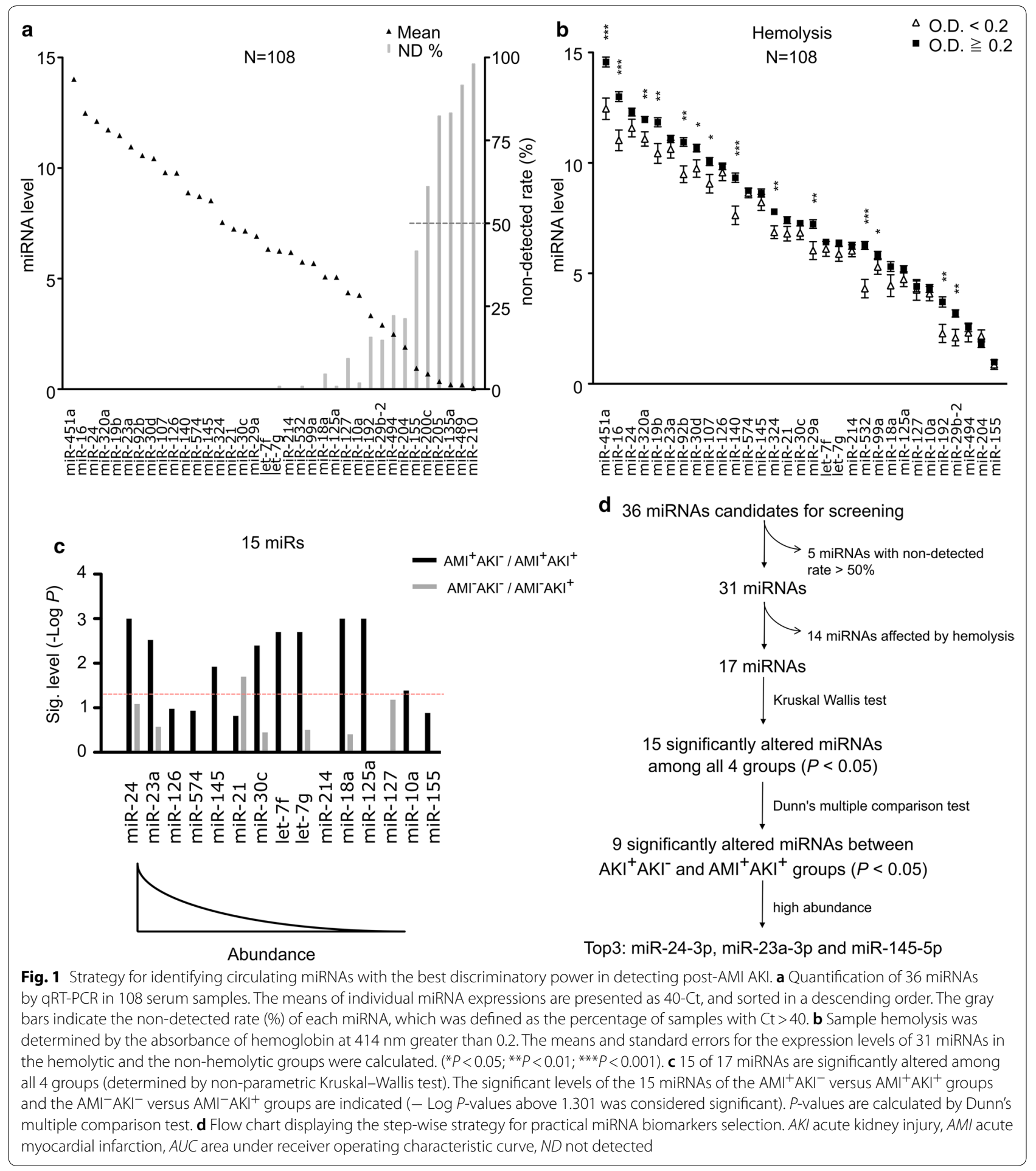

The miRNA panel (miR-24, miR-23a, miR-145) has the highest detection rate of post-AMI AKI

Based on the high sensitivity of miR-24 and miR-145 and the high specificity of miR-23a, the top two miRNAs (miR-24 and miR-23a) and the top three miRNAs
(miR-24, miR-23a and miR-145) were combined into panels, and used logistic regression analysis to evaluate the performance of these panels in detecting post-AMI AKI. As shown in Fig. 2b, the scores of the two-miRNA model and three-miRNA model were elevated in the $\mathrm{AMI}^{+} \mathrm{AKI}^{+}$ 

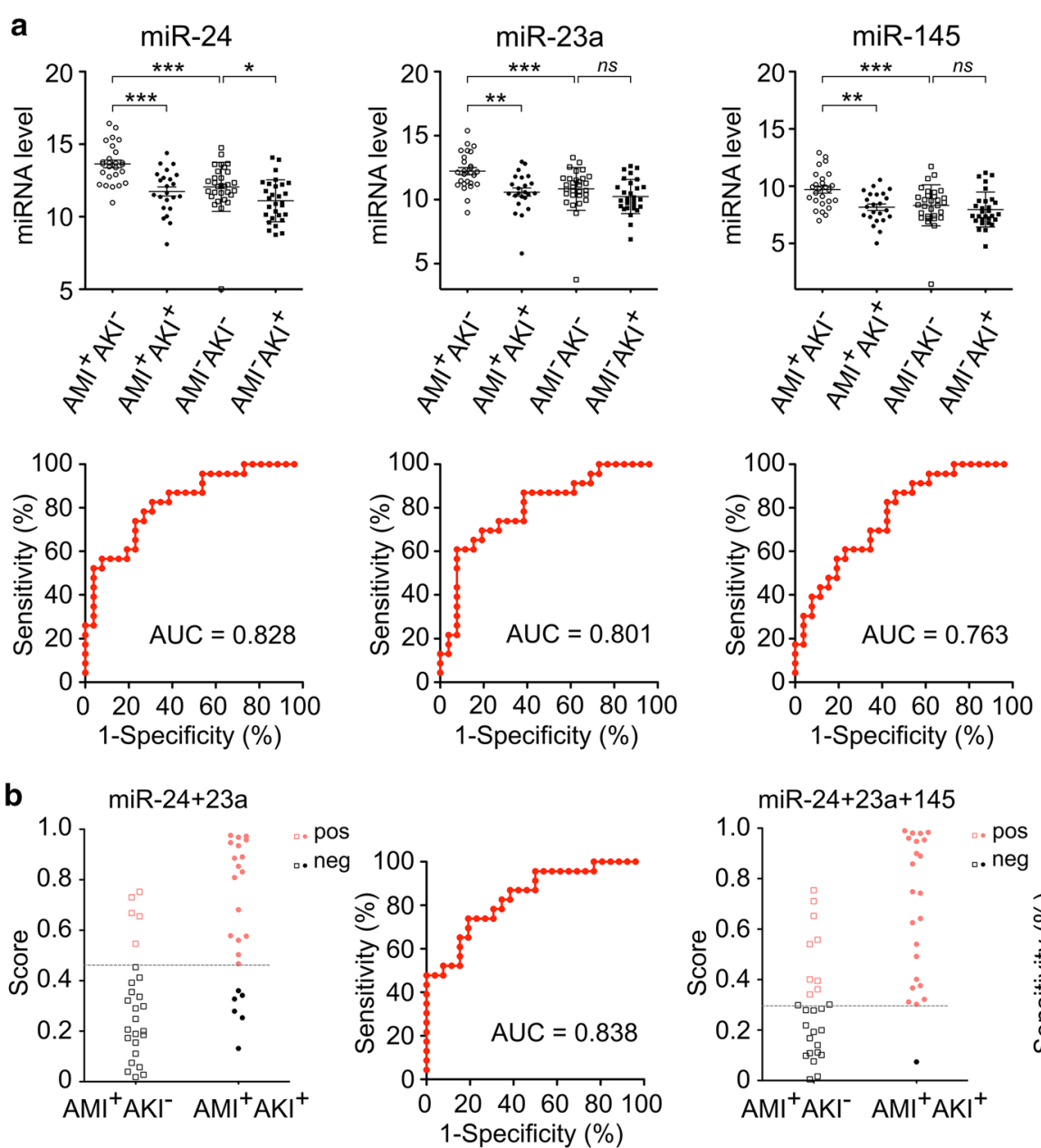

Fig. 2 The expression levels and diagnostic performances of miR-24, miR-23a and miR-145 in detecting post-AMI AKI. a Scatter plots representing the distributions for the expression levels of, miR-24, miR-23a and miR-145 in the AMI+ $\mathrm{AKI}^{-}$group (open squares) and the $\mathrm{AMI}^{+} \mathrm{AKI}{ }^{+} \mathrm{group}(\mathrm{full}$ circles). $P$-value was calculated by Mann-Whitney $U$ test. ( ${ }^{*} P<0.05 ;{ }^{* *} P<0.01 ;{ }^{* *} P<0.001 ; n$ s not significant). The ROC analysis and the AUC values were performed to discriminating between the $\mathrm{AMI}^{+} \mathrm{AKI}{ }^{-}$and the $\mathrm{AMI}{ }^{+} \mathrm{AKI}{ }^{+}$groups. $\mathbf{b}$ Scatter plots presenting the distributions of scores generated by logistic regression integrating the combined effects of miR-24+ miR-23a, or miR-24+ miR-23a + miR-145, in discriminating between the $\mathrm{AMI}^{+} \mathrm{AKI}{ }^{-}$and $\mathrm{AMI}^{+} \mathrm{AKI}{ }^{+}$groups. Scores ranging from 0 to 1 were generated for each sample and used to calculate ROC curves. The positive cases (red dots) in each group were determined according to the individual cut-off value obtained using Youden's index on the ROC curve. AKI acute kidney injury, AMI acute myocardial infarction, AUC area under receiver operating characteristic curve, ROC receiver-operating characteristic

group compared with those in the $\mathrm{AMI}^{+} \mathrm{AKI}^{-}$group. Using the two-miRNApanel with the cut-off value of 0.46 yielded a slightly higher AUC value of 0.838 , a sensitivity of $73.91 \%$ and a specificity of $80.77 \%$. Using the three-miRNA panel with the cut-off value of 0.30 yielded an AUC value of 0.853 , a remarkably increased sensitivity of $95.65 \%$ and a moderate specificity of $65.38 \%$ (Table 2 ). This three-miRNA signature detected 22 out of the 23 patients in the $\mathrm{AMI}^{+} \mathrm{AKI}^{+}$group and had the best performance for diagnosing post-AMI AKI.
miR-24, miR-23a and miR-145 are simultaneously up-regulated in AMI, but down-regulated in AKI

Our literature review indicated that miR-24, miR-23a and miR-145 were highly related to AKI and AMI (Additional file 1: Figure S2), and our experimental results showed that all three miRNAs were significantly downregulated in $\mathrm{AMI}^{+} \mathrm{AKI}^{+}$patients compared to those in $\mathrm{AMI}^{+} \mathrm{AKI}^{-}$ patients (Fig. 2a). miRNAs in the $\mathrm{AMI}^{-} \mathrm{AKI}^{+}$and $\mathrm{AMI}^{+} \mathrm{AKI}^{-}$groups were also examined. Comparing to the $\mathrm{AMI}^{-} \mathrm{AKI}^{-}$group (disease control), the $\mathrm{AMI}^{+} \mathrm{AKI}^{-}$group exhibited significantly higher expression levels of miR-24 
Table 2 Summarized diagnostic factors of the individual miRNAs, combined miRNA panels and serum NGAL for AKI

\begin{tabular}{|c|c|c|c|c|c|c|c|c|}
\hline \multirow[t]{2}{*}{ Variate } & \multicolumn{2}{|c|}{ Value (mean $\pm \mathrm{SE})$} & \multirow{2}{*}{$\begin{array}{l}\text { Fold-change } \\
\left(\mathrm{AMI}^{+} \mathrm{AKI}^{+} /\right. \\
\left.\mathrm{AMI}^{+} \mathrm{AKI}^{-}\right)\end{array}$} & \multirow[t]{2}{*}{$P$ (MWU test) } & \multirow[t]{2}{*}{ Cut-off } & \multirow[t]{2}{*}{ AUC $(95 \% \mathrm{Cl})$} & \multirow[t]{2}{*}{ Sensitivity (\%) } & \multirow[t]{2}{*}{ Specificity (\%) } \\
\hline & $\mathrm{AMI}^{+} \mathrm{AKI}^{-}$ & $\mathrm{AMI}^{+} \mathrm{AKI}^{+}$ & & & & & & \\
\hline miR-24 (Ct) & $13.64 \pm 0.26$ & $11.74 \pm 0.31$ & 0.27 & $<0.001$ & $<12.99$ & $0.828(0.711-0.941)$ & 82.61 & 69.23 \\
\hline $\operatorname{miR}-23 a(C t)$ & $12.23 \pm 0.28$ & $10.59 \pm 0.32$ & 0.32 & $<0.001$ & $<10.89$ & $0.801(0.676-0.927)$ & 60.87 & 92.31 \\
\hline miR-145 (Ct) & $9.70 \pm 0.31$ & $8.16 \pm 0.29$ & 0.34 & 0.002 & $<9.67$ & $0.763(0.631-0.894)$ & 86.96 & 53.85 \\
\hline sNGAL (ng/mL) & $64.32 \pm 7.50$ & $140.13 \pm 24.38$ & 2.18 & 0.008 & $>87.45$ & $0.735(0.578-0.892)$ & 63.16 & 84.62 \\
\hline $\begin{array}{l}\text { miR-24+miR-23a } \\
\text { (score) }\end{array}$ & $0.30 \pm 0.04$ & $0.66 \pm 0.06$ & 2.16 & $<0.001$ & $>0.46$ & $0.838(0.728-0.948)$ & 73.91 & 80.77 \\
\hline $\begin{array}{l}\text { miR-24+miR-145 } \\
\quad \text { (score) }\end{array}$ & $0.29 \pm 0.04$ & $0.67 \pm 0.06$ & 2.27 & $<0.001$ & $>0.54$ & $0.843(0.730-0.956)$ & 69.57 & 88.46 \\
\hline $\begin{array}{l}\text { miR-23a+miR- } \\
145 \text { (score) }\end{array}$ & $0.35 \pm 0.04$ & $0.61 \pm 0.05$ & 1.74 & $<0.001$ & $>0.58$ & $0.801(0.676-0.927)$ & 60.87 & 92.31 \\
\hline $\begin{array}{l}\text { miR-24+ miR- } \\
23 a+\text { miR-145 } \\
\text { (score) }\end{array}$ & $0.29 \pm 0.04$ & $0.67 \pm 0.06$ & 2.29 & $<0.001$ & $>0.30$ & $0.853(0.744-0.962)$ & 95.65 & 65.38 \\
\hline
\end{tabular}

$A K I$ cute kidney injury, $A M I$ acute myocardial infarction, $A U C$ area under receiver operating characteristic curve, $C l$ confidence interval, $C t$ cycle threshold, $M W U$ MannWhitney U, NGAL neutrophil gelatinase-associated lipocalin, SE standard error

(threefold, $P<0.001)$, miR-23a (2.62-fold, $P=0.001)$ and miR145 (2.6-fold, $P=0.004)$. In contrast, $\mathrm{AMI}^{-} \mathrm{AKI}^{+}$samples exhibited decreased expression levels of miR-24 (0.52-fold, $P=0.004)$, miR-23a (0.67-fold, $P=0.024)$ and miR-145 (0.78fold, $P=0.098)$. These data may explain the dramatic downregulation of these three miRNAs in AMI patients upon the development of AKI, and could suggest that theses miRNAs play relevant roles in AMI and AKI.

miR-24, miR-23a and miR-145 all target on TGF- $\beta$ signaling and apoptosis pathway

In order to demonstrate the possible functions involved in post-AMI AKI, our miRNA target analysis by MiRDB and TargetScan 7.1 databases identified 243, 548 and 291 predicted targets of miR-24, miR-23a and miR-145, respectively (Fig. 3a). Through Metacore pathway map analysis, the top five significantly enriched pathways for each miRNAare shown in Fig. 3b. Impressively, all of the three miRNAs are involved in two common pathways, TGF- $\beta$ signaling and apoptosis, in kidney injury. Among the 32 predicted target genes related to TGF- $\beta$ signaling and apoptosis pathway, 14 target genes (ACVR1B, APAF1, CASP7, FAS, LRP5, MAPK14, PDGFRB, PXN, SERPINE1/PAI1, SMAD2, SMAD3, TCF3, TGFBR2 and XIAP) had been experimentally proved by previous studies (Table 3). All these data support that the three miRNAs simultaneously regulate TGF- $\beta$ signaling and apoptosis pathway.

\section{Discussion}

In the present study, a thorough literature review was performed to select 36 miRNA candidates as potential biomarkers for post-AMI AKI. From among them, serum miR-24, miR-23a and miR-145 were found to be significantly down-regulated in $\mathrm{AMI}^{+} \mathrm{AKI}^{+}$patients and showed good discriminatory power (i.e., better than serum NGAL) in detecting in post-AMI AKI within $24 \mathrm{~h}$ of admission. Our logistic regression analysis showed that this three-miRNA panel exhibited better diagnostic performance than the individual miRNAs or a two-miRNA panel. Our target prediction and pathway analyses showed that these three miRNAs commonly regulate TGF- $\beta$ signaling and apoptosis. To our knowledge, this is the first report to identify a unique circulating miRNA signature for detecting post-AMI AKI at an early stage and demonstrate their pathogenic mechanisms.

Our results demonstrated that the three-miRNA panel had excellent performance in the early the diagnosis of post-AMI AKI. Over the last decade, NGAL has been known as one of the most promising and sensitive novel protein biomarkers for diagnosing AKI, with a reported AUC of 0.815 [24]. When NGAL was combined with KIM-1 and $N$-acetyl-beta-D-glucosaminidase (NAG), the AUC of the three-protein panel could reach 0.840 [25]. In our current study, using samples obtained within $24 \mathrm{~h}$ of admission, miR-24 alone (AUC $=0.828)$, miR-23a alone $(\mathrm{AUC}=0.801)$, miR-145 alone $(\mathrm{AUC}=0.763)$ and the three-miRNA panel $(\mathrm{AUC}=0.853)$ all had higher discriminatory abilities than serum NGAL (AUC=0.735) in diagnosing post-AMI AKI. Our data indicate that the three-miRNA panel is a promising diagnostic tool for post-AMI AKI.

To determine this signature for post-AMI AKI, three disease control groups were used from $\mathrm{CCU}$ patients $\left(\mathrm{AMI}^{-} \mathrm{AKI}^{-}, \mathrm{AMI}^{+} \mathrm{AKI}^{-}\right.$and $\left.\mathrm{AMI}^{-} \mathrm{AKI}^{+}\right)$rather than normal healthy individuals. Previous studies separately 


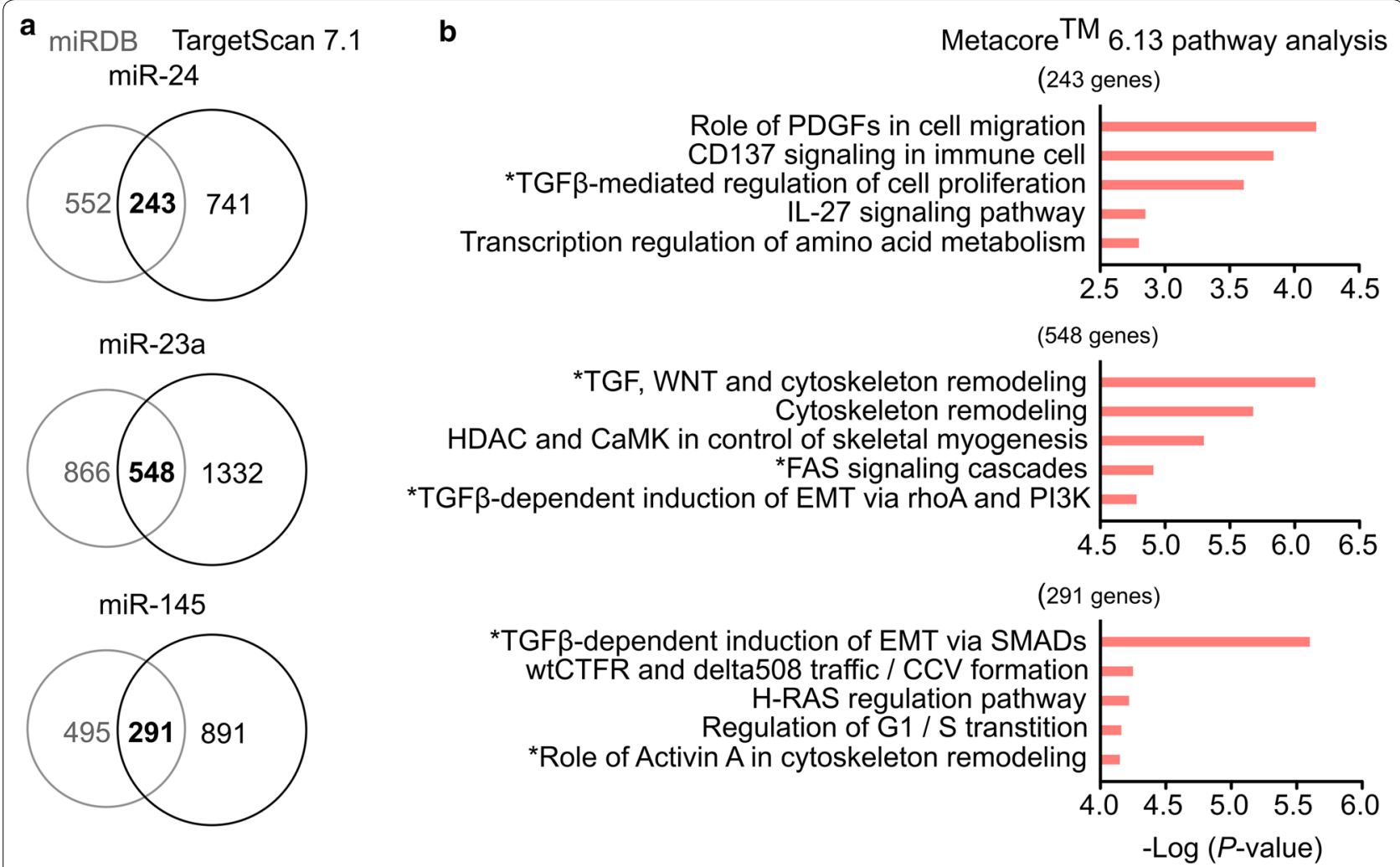

Fig. 3 Target prediction and pathway analysis of miR-24, miR-23a and miR-145 target genes. a Venn diagrams showing the potential miRNA targets predicted from TargetScan 7.1 and MiRDB. b The lists of intersecting genes were further examined by Metacore ${ }^{\text {TM }} 6.13$ pathway map analysis. The top five significantly enriched pathways are listed with their - Log $P$-values. TGF- $\beta$ - or apoptosis-associated pathways are marked with asterisks $\left(^{*}\right)$. TGF- $\beta$ transforming growth factor beta

discussed miRNA expression under AMI or AKI; however, both conditions have shared pathophysiologies including cell apoptosis, inflammation, and fibrosis. Some miRNAs were reported to be involved in both AMI and AKI, but it was not known whether they acted synergistically or antagonistically. The use of disease control groups allowed us to focus on the specific effect of AKI on miRNA expression among AMI patients, in turn enabling us to identify a circulating miRNA signature that is distinctive in diagnosing post-AMI AKI.

MiR-24, miR-23a and miR-145 had all been previously reported in AMI, and miR-24 and miR-145 had been implicated in AKI. MiR-24 was reportedly down-regulated in the plasma of critically ill patients with AKI [26]. Its tissue levels were up-regulated in a mouse model of renal ischemic reperfusion (I/R) injury and in renal transplant patients with prolonged cold ischemic time [27]. MiR-24 was shown to be induced by hypoxia inducible factor- 1 , and to thereby promote renal ischemic injury by stimulating apoptosis in endothelial and tubular epithelial cells. In vivo inhibition of miR-24 was shown to reduce tubular injury and improve renal function [27]. MiR-24 was found to be specifically enriched in cardiac endothelial cells in the infarct border zone after AMI [28]. Inhibition of endothelial miR-24 limited the myocardial infarct size of mice by preventing endothelial apoptosis and enhancing vascularity, thereby preserving cardiac function and survival. MiR-23a was found to be up-regulated in the plasma of patients with coronary artery diseases, and in an I/R-induced myocardial infarction model [29, 30]. MiR-23a was also shown to regulate cardiomyocyte apoptosis by inhibiting manganese superoxide dismutase [31]. MiR-145 is reportedly abundant in normal vascular smooth muscle cells, but down-regulated in injured or atherosclerotic vessels [32]. In contrast to patients with coronary artery diseases, the plasma levels of miR-145 were found to be increased in AMI patients; moreover, these levels were positively correlated with the infarct size [33]. Our current results agree with these previous reports and demonstrate for the first time that miR-23a is down-regulated in AKI. The distinctive expression pattern of these three miRNAs suggests that they may be functionally relevant. 
Table 3 Predicted and experimentally validated target genes of miR-24, miR-23a and miR-145 in TGF $\beta$ - and apoptosisrelated pathways

\begin{tabular}{|c|c|c|c|}
\hline \multicolumn{2}{|l|}{ Target } & \multicolumn{2}{|l|}{ miRNA } \\
\hline Gene symbol & Pathway & Prediction & Experiment \\
\hline ACVR1B & Role of activin A in cytoskeleton remodeling & miR-145 & $\operatorname{miR}-24(2), \operatorname{miR}-145(6,11)$ \\
\hline ACVR2A & Role of activin A in cytoskeleton remodeling & miR-145 & \\
\hline APAF1 & FAS signaling cascades & miR-23a & miR-23a $(17,20,23,25,28,34,42)$ \\
\hline CASP7 & FAS signaling cascades & miR-23a & $\operatorname{miR}-23 a(7,12,21)$ \\
\hline CFL2 & $\begin{array}{l}\text { TGF, WNT and cytoskeleton remodeling/TGF } \beta \text {-dependent induction of } \\
\text { EMT via rhoA and PI3 K }\end{array}$ & miR-23a & \\
\hline CHUK & $\begin{array}{l}\text { TGF, WNT and cytoskeleton remodeling/TGF } \beta \text {-dependent induction of } \\
\text { EMT via rhoA and PI3K }\end{array}$ & miR-23a & \\
\hline COL4A1 & TGF, WNT and cytoskeleton remodeling & miR-23a & \\
\hline FAS & FAS signaling cascades & miR-23a & miR-23a $(3,15,22)$ \\
\hline GSK3B & $\begin{array}{l}\text { TGF, WNT and cytoskeleton remodeling/TGF } \beta \text {-dependent induction of } \\
\text { EMT via rhoA and PI3K }\end{array}$ & miR-23a & \\
\hline LRP5 & TGF, WNT and cytoskeleton remodeling & miR-23a & miR-23a (36), miR-145 (38) \\
\hline MAP3K1 & FAS signaling cascades & miR-23a & \\
\hline MAP3K5 & FAS signaling cascades & miR-23a & \\
\hline MAPK14 & TGF $\beta$-mediated regulation of cell proliferation & miR-24 & miR-24 (1) \\
\hline MKL2 & TGF $\beta$-dependent induction of EMT via SMADs & miR-145 & \\
\hline MYL12B & TGF, WNT and cytoskeleton remodeling & miR-23a & \\
\hline NLK & TGF, WNT and cytoskeleton remodeling & miR-23a & \\
\hline PAK2 & FAS signaling cascades & miR-23a & \\
\hline PDGFRA & TGF $\beta$-mediated regulation of cell proliferation & miR-24 & \\
\hline PDGFRB & TGF $\beta$-mediated regulation of cell proliferation & miR-24 & miR-24 $(16,33)$ \\
\hline PDPK1 & TGF $\beta$-dependent induction of EMT via rhoA and PI3 K & miR-23a & \\
\hline PIK3C2A & $\begin{array}{l}\text { TGF, WNT and cytoskeleton remodeling/TGF } \beta \text {-dependent induction of } \\
\text { EMT via rhoA and PI3K }\end{array}$ & miR-23a & \\
\hline PIK3CB & $\begin{array}{l}\text { TGF, WNT and cytoskeleton remodeling/TGF } \beta \text {-dependent induction of } \\
\text { EMT via rhoA and PI3K }\end{array}$ & miR-23a & \\
\hline PPP1CB & TGF, WNT and cytoskeleton remodeling & miR-23a & \\
\hline PPP1R12A & TGF, WNT and cytoskeleton remodeling & miR-23a & \\
\hline PXN & Role of activin A in cytoskeleton remodeling & miR-145 & miR-145 (30) \\
\hline SERPINE1/PAI1 & TGF $\beta$-dependent induction of EMT via SMADs & miR-145 & miR-145 $(8,13)$ \\
\hline SMAD2 & $\begin{array}{l}\text { TGF } \beta \text {-dependent induction of EMT via SMADs/role of activin A in } \\
\text { cytoskeleton remodeling }\end{array}$ & miR-145 & miR-145 $(5,40)$ \\
\hline SMAD3 & $\begin{array}{l}\text { TGF } \beta \text {-dependent induction of EMT via SMADs/role of activin A in } \\
\text { cytoskeleton remodeling }\end{array}$ & miR-145 & miR-23a $(39,41)$, miR-145 $(14,27,29,31,37)$ \\
\hline TCF3 & TGF $\beta$-dependent induction of EMT via SMADs & $\operatorname{miR}-145$ & miR-24 (19) \\
\hline TGFBR2 & $\begin{array}{l}\text { TGF, WNT and cytoskeleton remodeling/TGF } \beta \text {-dependent induction of } \\
\text { EMT via rhoA and PI3K/TGF } \beta \text {-dependent induction of EMT via SMADs }\end{array}$ & miR-23a, miR-145 & miR-145 $(18,26,40)$ \\
\hline TJP1 & TGF $\beta$-dependent induction of EMT via rhoA and PI3K & miR-23a & \\
\hline XIAP & TGF, WNT and cytoskeleton remodeling/FAS signaling cascades & miR-23a & miR-23a $(4,9,35)$, miR-24 $(10,24,32)$ \\
\hline
\end{tabular}

The references are listed in Additional file 2: Supplementary references

Our bioinformatic study found that miR-24, miR-23a and miR-145 were commonly associated with TGF- $\beta$ signaling and apoptosis. TGF- $\beta$ is well recognized as a key mediator of renal fibrosis [34]. Evidence also showed that TGF- $\beta$ signaling participates in kidney injury. During AKI, tubular epithelial cells can produce TGF- $\beta$ and fractalkines to promote inflammation and kidney injury [35]. Deletion the TGF- $\beta$ type II receptor in the proximal tubules could attenuate tubular apoptosis and reduce renal injury in a mouse model, suggesting that TGF- $\beta$ signaling is pro-apoptotic in this setting [36]. In a transgenic mouse model, activation of TGF- $\beta$ signaling in 
the tubular epithelium alone was sufficient to cause AKI [37]. Tubular cell apoptosis, which is well recognized as the hallmark of AKI, involves intrinsic, extrinsic and endoplasmic reticulum stress pathways [38]. Previous studies showed that the three miRNAs selected in the present work can target TGF- $\beta$ signaling and apoptosis by regulating Furin, SMAD4, Bim, Bax, FOXO3, and SP1. Based on the bioinformatic analysis and previous experimental studies, a network model was proposed (Fig. 4) in which the three selected miRNAs suppress the expressions of TGF- $\beta$ signaling components (Furin, TGFBR2 and SMADs), the transcription factors (Foxo3 and SP1), and apoptosis-related molecules (Bim, Bax, Caspase 7, Apaf-1 and Fas receptor). Taken together, the three selected miRNAs not only have the potential to serve as early biomarkers for detecting post-AMI AKI, but may also contribute to post-AMI AKI pathogenesis by regulating TGF- $\beta$ signaling and apoptosis to promote renal injury and fibrosis. The biological relevance and therapeutic potential of these three miRNAs in AKIare worthy of further study.

\section{Limitations}

The study population was relatively small and not enough to evaluate the correlation of the three selected miRNAs with the severity of AKI or the long-term clinical outcome. Further investigation is needed to validate the accuracy and clinical utility of the three-miRNA panel to serve as an early detection tool of AKI following AMI or other cardiorenal syndromes.

\section{Conclusion}

In conclusion, for the first time, this study identified a unique circulating miRNA signature (miR-24, miR-23a and miR-145) that enables the early detection of postAMI AKI in human. These three miRNAs target TGF- $\beta$

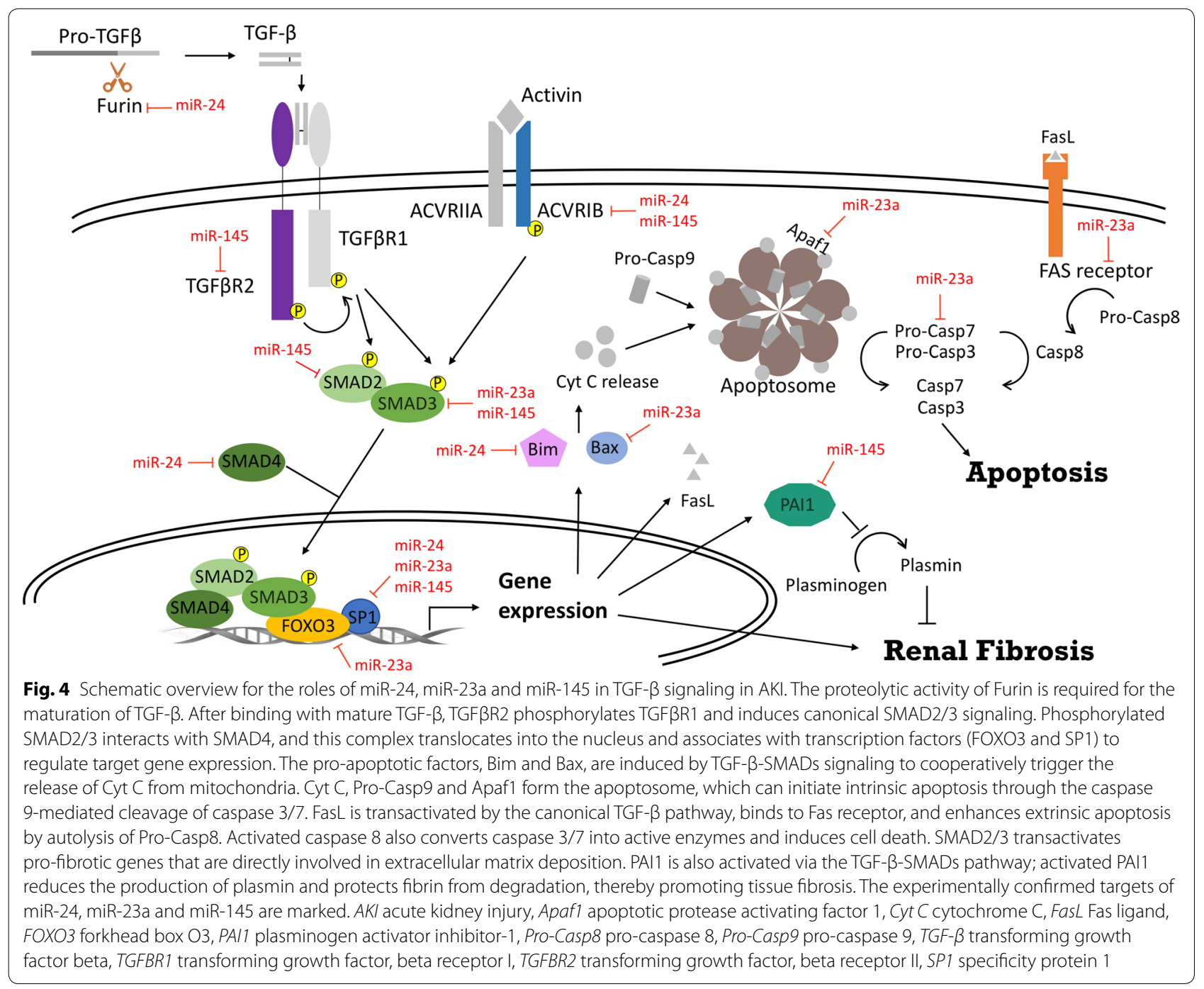


signaling and apoptosis, and may be involved in renal injury and fibrosis in post-AMI AKI pathogenesis.

\section{Additional files}

Additional file 1: Figure S1. Study design. Figure S2. Summary of total reference numbers, disease distribution and sample type analysis of the miRNA candidates. Table S3. Kruskal-Wallis test and post hoc Dunn's multiple comparisons of the 17 miRNAs among all groups $(N=108)$.

Additional file 2: Tables S1, S2. Literature review of 119 research articles related to AKI, AMI and cardiovascular diseases. Supplementary references. Supplementary references of Additional file 1: Table S3.

\section{Abbreviations}

ACVR1B: activin A receptor type 1B; AKl: acute kidney injury; AKIN: Acute Kidney Injury Network; AMI: acute myocardial infarction; APAF1: apoptotic protease activating factor 1; AUC: area under receiver operating characteristic curve; CASP7: caspase 7; CCU: coronary care unit; CKD: chronic kidney disease; CVD: cardiovascular disease; ELISA: enzyme-linked immunosorbent assay; FasL: Fas ligand; FOXO3: forkhead box O3; I/R: ischemic reperfusion; KDIGO Kidney Disease Improving Global Outcomes; KIM-1: kidney injury molecule-1; LRP5: low-density lipoprotein-related receptors 5; MAPK14: mitogen-activated protein kinase 14; miRNA: microRNA; NAG: N-acetyl-beta-D-glucosaminidase; ND: not detected; NGAL: neutrophil gelatinase-associated lipocalin; PAI1: plasminogen activator inhibitor-1; PCR: polymerase chain reaction; PDGFRB: platelet-derived growth factor receptor beta; Pro-Casp8: pro-caspase 8; ProCasp9: pro-caspase 9; PXN: paxillin; RIFLE: Risk, Injury, Failure, Loss of kidney function, and End-stage kidney disease; ROC: receiver-operating characteristic; SCr: serum creatinine; SERPINE1: serpin family E member 1; SP1: specificity protein 1 ; TCF3: transcription factor 3 ; TGF- $\beta$ : transforming growth factor beta; TGFBR1: transforming growth factor, beta receptor I; TGFBR2: transforming growth factor, beta receptor II; XIAP: X-linked inhibitor of apoptosis protein.

\section{Authors' contributions}

PCF, CCC: data analysis and interpretation, drafting the article; CCP, CY, LJC: data analysis and interpretation; $\mathrm{CHC}, \mathrm{CHY}$ : data collection; YCC, CWY: critical revision of the article; YSC: conception or design of the work, final approval of the version to be published; PHC: conception or design of the work, Final approval of the version to be published. All authors read and approved the final manuscript.

\section{Author details}

${ }^{1}$ Department of Nephrology, Kidney Research Center, Chang Gung Memorial Hospital, Linkou Medical Center, Chang Gung University College of Medicine, No. 5 Fusing Street, Gueishan Dist., Taoyuan City 333, Taiwan, ROC. ${ }^{2}$ Graduate Institute of Clinical Medical Sciences, Chang Gung University, No. 5 Fusing Street, Gueishan Dist., Taoyuan City 333, Taiwan, ROC. ${ }^{3}$ Molecular Medicine Research Center, Chang Gung University, No 259 Wen-Hwa 1st Road, Kwei-Shan, Taoyuan City 33302, Taiwan, ROC. ${ }^{4}$ Department of Colorectal Surgery, Chang Gung Memorial Hospital, No 259 Wen-Hwa 1st Road, Kwei-Shan, Linkou, Taoyuan City 33302, Taiwan, ROC. ${ }^{5}$ Department of Cardiology, Chang Gung Memorial Hospital, Chang Gung University College of Medicine, No. 5 Fusing Street, Gueishan Dist., Taoyuan City 333, Taiwan, ROC. ${ }^{6}$ Department of Nephrology, Chang Gung Memorial Hospital, Keelung Branch, Chang Gung University College of Medicine, No. 222, Maijin Rd., Anle Dist., Keelung City 20401, Taiwan, ROC. 7 Department of Cardiology, Chang Gung Memorial Hospital, Chang Gung University College of Medicine, 199 Tung Hwa North Road, Taipei 105, Taiwan.

\section{Acknowledgements}

The authors thank the participants of the Kidney Research Center of Chang Gung Memorial Hospital, Linkou, Taiwan; and Yuka Chu's critical reading. All authors have read the journal's authorship agreement and reviewed and approved the manuscript.

\section{Competing interests}

The authors declare that they have no competing interests.

\section{Availability of data and materials}

All the data generated or analysed during this study are included in this published article.

\section{Consent for publication}

Not applicable.

\section{Ethics approval and consent to participate}

The study protocol was approved by the local institutional review board, and informed consents were obtained from all participants.

\section{Funding}

This research is supported by research grants from the Chang Gung Medical Foundation (CORPG3H0091, CIRPG3B0042, CIRPG3B0043, CIRPD3B0012, CIRPD3B0013 and CMRPG5G0111), and the Ministry of Science and Technology of Taiwan (103-2314-B-182A-018-MY3, 106-2314-B-182A-118-MY3).

\section{Publisher's Note}

Springer Nature remains neutral with regard to jurisdictional claims in published maps and institutional affiliations.

Received: 15 November 2018 Accepted: 23 April 2019

Published online: 30 April 2019

\section{References}

1. Bellomo R, Ronco C, Kellum JA, Mehta RL, Palevsky P, Acute Dialysis Quality Initiative w. Acute renal failure-definition, outcome measures, animal models, fluid therapy and information technology needs: the Second International Consensus Conference of the Acute Dialysis Quality Initiative (ADQI) Group. Crit Care. 2004;8(4):R204-12.

2. Bruetto RG, Rodrigues FB, Torres US, Otaviano AP, Zanetta DM, Burdmann EA. Renal function at hospital admission and mortality due to acute kidney injury after myocardial infarction. PLoS ONE. 2012;7(4):e35496.

3. Fox CS, Muntner P, Chen AY, Alexander KP, Roe MT, Wiviott SD. Short-term outcomes of acute myocardial infarction in patients with acute kidney injury: a report from the national cardiovascular data registry. Circulation. 2012;125(3):497-504.

4. Parikh CR, Coca SG, Wang Y, Masoudi FA, Krumholz HM. Long-term prognosis of acute kidney injury after acute myocardial infarction. Arch Intern Med. 2008;168(9):987-95.

5. Rodrigues FB, Bruetto RG, Torres US, Otaviano AP, Zanetta DM, Burdmann EA. Incidence and mortality of acute kidney injury after myocardial infarction: a comparison between KDIGO and RIFLE criteria. PLOS ONE. 2013;8(7):e69998.

6. Marenzi G, Cosentino N, Bartorelli AL. Acute kidney injury in patients with acute coronary syndromes. Heart. 2015;101(22):1778-85.

7. Amin AP, Spertus JA, Reid KJ, Lan X, Buchanan DM, Decker C, et al. The prognostic importance of worsening renal function during an acute myocardial infarction on long-term mortality. Am Heart J. 2010;160(6):1065-71.

8. Chawla LS, Kimmel PL. Acute kidney injury and chronic kidney disease: an integrated clinical syndrome. Kidney Int. 2012;82(5):516-24.

9. Coca SG, Singanamala S, Parikh CR. Chronic kidney disease after acute kidney injury: a systematic review and meta-analysis. Kidney Int. 2012;81(5):442-8

10. Mehta RL, Kellum JA, Shah SV, Molitoris BA, Ronco C, Warnock DG, et al. Acute Kidney Injury Network: report of an initiative to improve outcomes in acute kidney injury. Crit Care. 2007;11(2):R31.

11. Group KDIGOKAKIW. KDIGO clinical practice guideline for acute kidney injury. Kidney Int Suppl. 2012;2:1-138.

12. Mishra J, Ma Q, Prada A, Mitsnefes M, Zahedi K, Yang J, et al. Identification of neutrophil gelatinase-associated lipocalin as a novel early urinary biomarker for ischemic renal injury. J Am Soc Nephrol. 2003;14(10):2534-43. 
13. Han WK, Bailly V, Abichandani R, Thadhani R, Bonventre JV. Kidney Injury Molecule-1 (KIM-1): a novel biomarker for human renal proximal tubule injury. Kidney Int. 2002;62(1):237-44.

14. Krol J, Loedige I, Filipowicz W. The widespread regulation of microRNA biogenesis, function and decay. Nat Rev Genet. 2010;11(9):597-610.

15. Fan PC, Chen CC, Chen YC, Chang YS, Chu PH. MicroRNAs in acute kidney injury. Hum Genomics. 2016;10(1):29.

16. Sun T, Dong YH, Du W, Shi CY, Wang K, Tariq MA, et al. The role of microRNAs in myocardial infarction: from molecular mechanism to clinical application. Int J Mol Sci. 2017. https://doi.org/10.3390/ijms18040745 (pii:E745).

17. Rupaimoole R, Slack FJ. MicroRNA therapeutics: towards a new era for the management of cancer and other diseases. Nat Rev Drug Discov. 2017;16(3):203-22.

18. Thygesen K, Alpert JS, White HD, Joint ESCAAHAWHFTFftRoMI. Universal definition of myocardial infarction. Eur Heart J. 2007;28(20):2525-38.

19. Chang PY, Chen CC, Chang YS, Tsai WS, You JF, Lin GP, et al. MicroRNA-223 and microRNA-92a in stool and plasma samples act as complementary biomarkers to increase colorectal cancer detection. Oncotarget. 2016:7(9):10663-75.

20. Kirschner MB, Kao SC, Edelman JJ, Armstrong NJ, Vallely MP, van Zandwijk $\mathrm{N}$, et al. Haemolysis during sample preparation alters microRNA content of plasma. PLoS ONE. 2011;6(9):e24145.

21. Shah JS, Soon PS, Marsh DJ. Comparison of methodologies to detect low levels of hemolysis in serum for accurate assessment of serum microRNAs. PLoS ONE. 2016;11(4):e0153200.

22. Wu CS, Lin FC, Chen SJ, Chen YL, Chung WJ, Cheng Cl. Optimized collection protocol for plasma microRNA measurement in patients with cardiovascular disease. Biomed Res Int. 2016;2016:2901938.

23. Kirschner MB, Edelman JJ, Kao SC, Vallely MP, van Zandwijk N, Reid G. The impact of hemolysis on cell-free microRNA biomarkers. Front Genet. 2013;4:94.

24. Haase M, Bellomo R, Devarajan P, Schlattmann P, Haase-Fielitz A, Group NM-al. Accuracy of neutrophil gelatinase-associated lipocalin (NGAL) in diagnosis and prognosis in acute kidney injury: a systematic review and meta-analysis. Am J Kidney Dis. 2009;54(6):1012-24.

25. Han WK, Wagener G, Zhu Y, Wang S, Lee HT. Urinary biomarkers in the early detection of acute kidney injury after cardiac surgery. Clin J Am Soc Nephrol. 2009;4(5):873-82.
26. Lorenzen JM, Kielstein JT, Hafer C, Gupta SK, Kumpers P, Faulhaber-Walter $\mathrm{R}$, et al. Circulating miR-210 predicts survival in critically ill patients with acute kidney injury. Clin J Am Soc Nephrol. 2011;6(7):1540-6.

27. Lorenzen JM, Kaucsar T, Schauerte C, Schmitt R, Rong S, Hubner A, et al. MicroRNA-24 antagonism prevents renal ischemia reperfusion injury. J Am Soc Nephrol. 2014;25(12):2717-29.

28. Fiedler J, Jazbutyte V, Kirchmaier BC, Gupta SK, Lorenzen J, Hartmann D, et al. MicroRNA-24 regulates vascularity after myocardial infarction. Circulation. 2011;124(6):720-30.

29. Han H, Qu G, Han C, Wang Y, Sun T, Li F, et al. MiR-34a, miR-21 and miR-23a as potential biomarkers for coronary artery disease: a pilot microarray study and confirmation in a 32 patient cohort. Exp Mol Med. 2015:47:e138.

30. Wang S, He W, Wang C. MiR-23a regulates the vasculogenesis of coronary artery disease by targeting epidermal growth factor receptor. Cardiovasc Ther. 2016;34(4):199-208.

31. Long B, Gan TY, Zhang RC, Zhang YH. miR-23a regulates cardiomyocyte apoptosis by targeting manganese superoxide dismutase. Mol Cells. 2017:40(8):542-9.

32. Guo C, Deng Y, Liu J, Qian L. Cardiomyocyte-specific role of miR-24 in promoting cell survival. J Cell Mol Med. 2015;19(1):103-12.

33. Meder B, Keller A, Vogel B, Haas J, Sedaghat-Hamedani F, Kayvanpour E, et al. MicroRNA signatures in total peripheral blood as novel biomarkers for acute myocardial infarction. Basic Res Cardiol. 2011;106(1):13-23.

34. Bottinger EP.TGF-beta in renal injury and disease. Semin Nephrol. 2007;27(3):309-20.

35. Bonventre JV, Zuk A. Ischemic acute renal failure: an inflammatory disease? Kidney Int. 2004;66(2):480-5.

36. Gewin L, Vadivelu S, Neelisetty S, Srichai MB, Paueksakon P, Pozzi A, et al. Deleting the TGF-beta receptor attenuates acute proximal tubule injury. J Am Soc Nephrol. 2012;23(12):2001-11.

37. Gentle ME, Shi S, Daehn I, Zhang T, Qi H, Yu L, et al. Epithelial cell TGFbeta signaling induces acute tubular injury and interstitial inflammation. J Am Soc Nephrol. 2013;24(5):787-99.

38. Linkermann A, Chen G, Dong G, Kunzendorf U, Krautwald S, Dong Z. Regulated cell death in AKI. J Am Soc Nephrol. 2014;25(12):2689-701.
Ready to submit your research? Choose BMC and benefit from:

- fast, convenient online submission

- thorough peer review by experienced researchers in your field

- rapid publication on acceptance

- support for research data, including large and complex data types

- gold Open Access which fosters wider collaboration and increased citations

- maximum visibility for your research: over 100M website views per year

At BMC, research is always in progress.

Learn more biomedcentral.com/submissions 